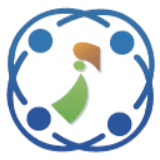

\title{
ECG Signal Enhancement through Subband Adaptive Soft Thresholding and EMD for Efficient Cardiac Arrhythmia Analysis
}

\author{
Gopisetty Ramesh ${ }^{1 *}$ \\ Donthi Satyanarayana ${ }^{2}$ \\ Maruvada Sailaja ${ }^{3}$ \\ ${ }^{1}$ Department of Electronics and Communication Engineering, \\ Jawaharlal Nehru Technological University, Kakinada, Andhra Pradesh, 533003, India \\ ${ }^{2}$ Department of Electronics and Communication Engineering, \\ Rajeev Gandhi Memorial College of Engineering \& Technology, Nandyal, Andhra Pradesh, 518501, India \\ ${ }^{3}$ Department of Electronics and Communication Engineering, \\ Jawaharlal Nehru Technological University, Kakinada, Andhra Pradesh, 533003, India \\ * Corresponding author's Email: gopisettyramesh1980@gmail.com
}

\begin{abstract}
Among various Bio-medical signals, Electrocardiogram (ECG) is most important signal to diagnose Cardiac Arrhythmia (CA). However the noises added during ECG signal acquisition makes the system less accurate in CA detection. This paper presents a new ECG denoising approach based on noise reduction aspects in Discrete Wavelet Transform (DWT) and Empirical Mode Decomposition (EMD) domains. The main objective to use DWT is to remove the high frequency noise components like EMG noise which leads to significant cropping in QRS complexes and also lead to distortions at the beginning and ending of QRS complexes. This approach also designs an adaptive threshold which considers the average noise and signal powers to remove the noise form ECG signal. The segmentation of ECG signal involves to focus only on the noise dominant components such that the overall complexity also in the control. Further the EMD preserves the QRS complexes information by adapting the adaptive thresholding for the Intrinsic Mode Functions (IMFs) after finding the noise dominant IMFs from signal dominant IMFs. Simulation results are carried out through MIT-BIH Arrhythmia database and the performance is evaluated with respect to the performance metrics like Mean Square Error and Signal to Noise Ratio. The proposed approach provides better results compared to the conventional state-of the-art techniques.
\end{abstract}

Keywords: ECG, DWT, EMD, QRS Complex, Thresholding, MIT-Arrhythmia database, MSE, SNR.

\section{Introduction}

Cardiovascular diseases (CVDs) remain the leading cause of non-communicable deaths worldwide. According to the latest World Health Organization (WHO) report, about 17.5 million people died from CVDs in 2012, accounting for $30 \%$ of all global deaths. The incidence of CVD deaths is predicted to rise to 23 million by 2030 [1]. Furthermore, the costs for CVD-related treatment including medication are substantial. The CVDrelated cost in the low- and middle-income countries over the period 2011-2025 is estimated approximately 3.8 trillion U.S. dollars [2]. Many of these deaths and associated economic losses can be avoided by early detection and monitoring of patients' cardiac function. Electrocardiogram (ECG) is the standard and most efficient tool for CVD diagnosis [3], which captures the electrical activity of the heart from a human body surface, providing important information on cardiac functional abnormalities. Since the unique morphological characteristics of ECG, i.e., P-QRS-T complex, they have more significance in medical field compared to other biomedical signals. Just by analyzing these varying characteristics of ECG signal, many cardiac diseases can be diagnosed visually. But the noise presence in the ECG signals will affect the visual diagnosis severely and results in the wrong diagnosis in the feature extraction of different application like emotion prediction, stress 
measurement and computer human interface etc. The external noises (Power line interference, Muscle noise, Baseline wander nose etc.) added in the ECG signal during its recording crops the peaks in the QRS complex of the ECG signal. Further these noises also makes the ECG signal broaden in spectrum by introducing some distortions at the starting and ending of QRS complex. Particularly the muscle noise (EMG noise) is dominant at high frequencies and overlaps with the spectrum of ECG, specifically with the QRS complex spectrum. In such case, feature extraction from an ECG signal to perform the CVD diagnosis becomes difficult. In earlier, several approaches are proposed to perform noise filtering in ECG and to extract the efficient morphological feature set from ECG signals [4 - 8]

Generally the noise filtering approaches removes the extra noises from the ECG signal though thresholding technique. However a thresholding without an adaptive nature causes a non-recoverable damage to the signal. According to the related work, the denoising methods based on wavelet and Empirical Mode Decomposition (EMD) is found to be more effective in reducing the noise from ECG signal. However, processing the noise contaminated ECG signal through any of EMD or wavelet alone will result in inadequate denoising in the concern of reliable clinical applications [9]. If the signal is processed in the EMD domain alone, the initial IMFs are assumed to be excluded because the high frequency noise information is maximally stored in the initial IMFs. However this causes a severe damage to the signal quality. Further, processing of the signal purely in wavelet domain results in high computational overhead and also there is a need to design an adaptive threshold for every subband obtained through wavelet decomposition. Depends on the importance of wavelet coefficient the threshold needs to be varying such that the required information will be preserved in the thresholded coefficient. In the case of High frequency noise like EMG, an extra care has to take in the threshold design.

This paper proposes a novel hybrid filtering mechanism to remove the noise components from ECG signal followed QRS complex information preservation. Both the EMD and wavelet domain are accomplished in the proposed approach. Initially the noise contaminated ECG signal is processed through wavelet domain and an adaptive thresholding is done to remove the noise components only from high frequency sub bands. Further the partially noise filtered ECG signal is processed in the EMD domain to filter out the remaining noise. Here the wavelet thresholding considers the average signal variance to separate the high frequency subband into signaldominant and noise-dominant frequency bins and then an average noise power is used to perform thresholding of noise-dominant frequency bins only. Extensive simulations carried out over the proposed approach illustrate the performance of proposed approach.

Rest of the paper is organized as follows; section 2 illustrates the details of literature survey. Section 3 gives the details of proposed approach. The performance evaluation is carried out in 4 section and a comparative analysis is also illustrated. Finally section 5 concludes the paper.

\section{Literature survey}

In earlier, numerous methods are proposed to denoise the ECG signals based on filter banks, adaptive filtering [7, 8], Neural Networks (NNs), independent component analysis (ICA) [6, 12], principal component analysis (PCA) [10], Kernel PCA [11], wavelet transform [14 - 16], Empirical Mode Decomposition [5, 9, 13] and hybrid approaches by involving both EMD and wavelet transform $[17,18]$.

The approaches based of PCA, ICA and Neural Networks removes the in-band noise by discarding its corresponding components after establishing a statistical model for the ECG signal and noise. Though the PCA, ICA and NNs based denoising mechanisms are more powerful in denoising the inband noise of the ECG signal, the statistical model derived is extremely sensitive to the small changes in either signal or noise unless the corresponding ECG beats trained through the global set of features related to those changes. A specific drawback of ICA is the determination of order of Independent Components (ICs). Hence a visual inspection is required to process the signal through ICA which is an undesirable for ECG based clinical applications [19]. In the case of an adaptive filtering based ECG denoising, there is an additional complexity due to the recording of an additional reference signal along with ECG [20].

Compared with these approaches, the EMD and wavelet based denoising [24] approaches are found to be more effective in suppressing the noise from noise contaminated ECG signal. Since the recorded ECG is a mixture of signal and noise, thresholding in the frequency domain through an adaptive threshold results the noise free signal and also a qualitative signal having the required information preserved. In this approaches design of threshold plays an important role in the performance achievement. Since the ECG is a non-stationary 
signal, a linear threshold wont produces a better result. A novel thresholding technique is proposed in [4] based on modified S-median filter to filter out the noises from ECG signal. This method accomplished a 6th order Symlet wavelet filter and the input signal is decomposed to 8th level and then the modified S-median thresholding is applied over the obtained subbands. The performance evaluation is carried out through the "MIT-BIH noise stress test database". The main reason behind the Symlet is its scaling function's closer nature to the shape of ECG signal. An adaptive dual threshold filter is proposed in [16] based on Discrete Wavelet Transform to denoise a noise corrupted ECG signal. The main objective is to deal with the EMG noises, the high frequency noises and power line interferences that could disturb the ECG signal. The complete method is processed in three steps namely, the DWT decomposition, Adaptive dual threshold filtering and highest peaks correction. Another form of wavelet, stationary wavelet transform (SWT) is also used for ECG signal denoising. Smital et al., [21] and Phinyomark et al., [23] considered "wavelet wiener filtering (WWF)" [22] as a filter bank to estimate the noise free signal there by the non-stationary broadband myopotentials (EMG) will be filtered out more effectively. The dyadic stationary wavelet transform (SWT) is accomplished in the wiener filter to estimate the noise free signal. The main aim is to find an appropriate filter bank and its control parameters with respect to the obtained Signal-toNoise Ratio (SNR). The DWT is more effective in removing the high-frequency noise but requires a larger decomposition levels to de-noise the low frequency components. Thresholding low-frequency components in the DWT domain often results in the loss of significant information. To overcome this drawbacks, Partiksingh et al., [25] proposed 2-level DWT decomposition to obtain low and high frequency subbands. Then the high frequency noise is removed at both levels through thresholding and the noise in the low frequency region is removed through Non-Local Means (NLM) estimation of Level-2 approximation coefficient.

Though the wavelet thresholding (WT) based ECG denoising approaches are more efficient, the computational complexity is observed to be high due to the accomplishment of thresholding on the both high-frequency and low-frequency subbands. Further the EMD based denoising approaches tries to exclude initial IMFs because most of the noise information is present in initial IMFs only. Further after excluding some initial IMFs, the signal is reconstructed which will be noise free. However the main problem is to select the number of IMFs which have to consider for reconstruction. If EMD is accomplished with wavelet thresholding, most of the high frequency noise content will be filtered through DWT thresholding and further the EMD preserves the QRS complex information even form the initial IMFs. Based on this aspect some approaches are proposed by combining EMD with Wavelet Thresholding.

Unlike to the earlier developed approaches based on EMD that removes the initial intrinsic mode functions (IMFs) to filter the noise in ECG signal, the method proposed in [18] by Kabir et al., considered the initial IMFs also and performed a windowing technique over these IMFs to preserve the QRS complex information and also to remove the external noises from ECG signal. This approach attained efficient results in the noise filtering and also in the preservation of QRS complex information. Further the output signal of EMD is transformed through Discrete Wavelet Transform, where an adaptive soft thresholding technique is accomplished to remove the noise based on the advantageous properties of DWT, i.e., energy preservation even in the presence of noise and makes the reconstructed signal rich in quality. Though this approach achieved a better performance, the adaptive soft threshold considered in DWT domain discards the signal components also. This degradation results in the loss of the signal quality.

To overcome this issue, a novel approach is proposed in this paper by combining the wavelet thresholding with EMD. Initially the noise contaminated ECG signal is decomposed into lowfrequency and high frequency subbands through 2level DWT. Further the low-frequency (approximate) subband confidents are excluded from the thresholding and only high frequency (detailed) subband coefficients are processed for adaptive soft thresholding. Instead of applying the adaptive soft thresholding over the entire high frequency subbands at both levels, they are segmented into frames and then into frequency bins. Further they are categorized into noise-dominant and signal dominant frequency bins and the noise dominant frequency bins are subjected to adaptive thresholding. In the EMD based denoising, instead of discarding the initial IMFs, they are also considered to preserve the QRS complex information.

\section{Proposed approach}

In this section, the complete details of proposed approach are illustrated. The accomplishment of 


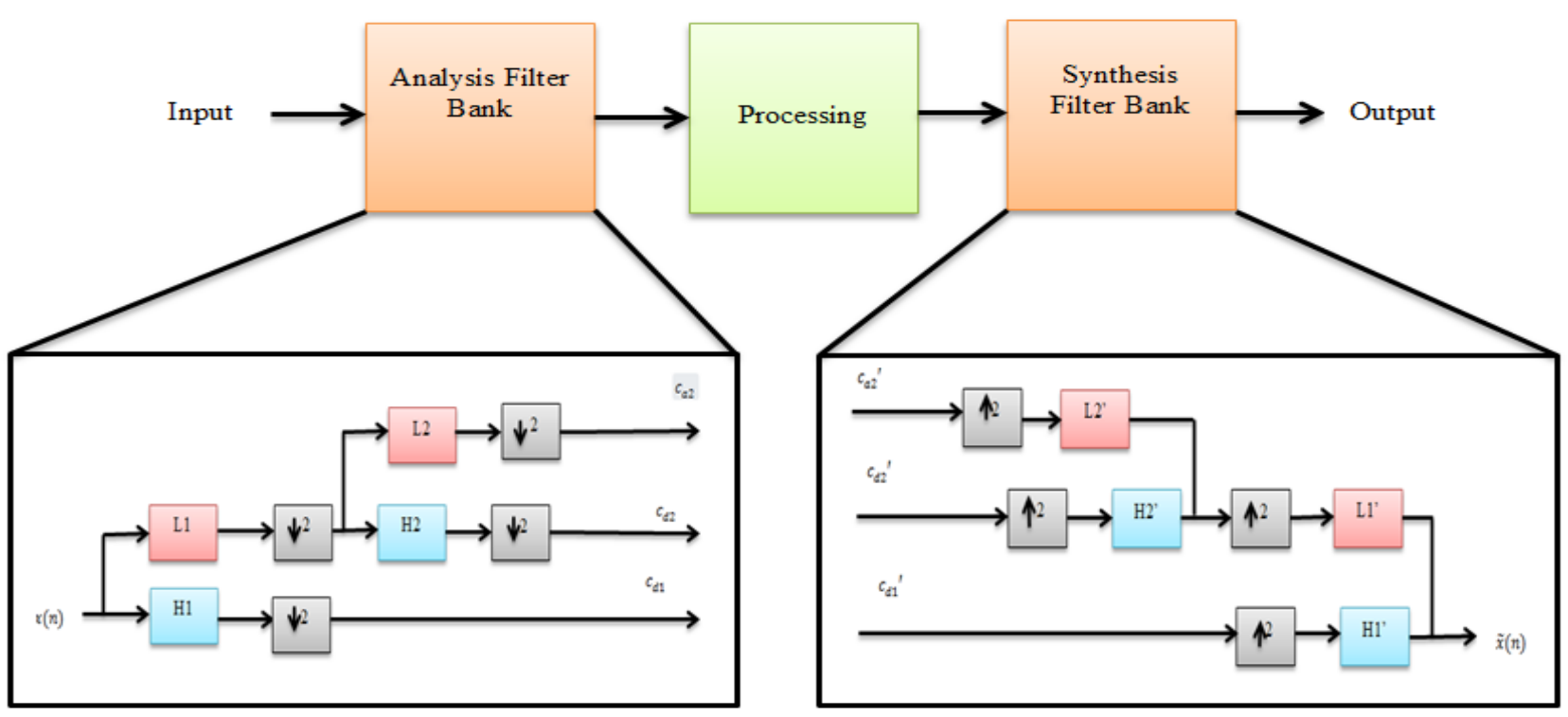

Figure.1 General architecture of DWT processing

proposed approach is carried out in two phases, one is DWT based soft thresholding and another is EMD based denoising. This approach is a hybrid mechanism in which the input ECG signal is analyzed in both time and transformation domain to achieve better results. In the first phase, the DWT based soft thresholding tries to filter out the noise components from all the noise contaminated frequencies and the EMD based denoising mainly focused in the preservation of QRS complexes which are more important in the analysis of Cardiovascular disease. Since the rate of change in the information is very high in QRS complexes compared to the other parts of ECG signal, the main objective is kept towards the preservation. The complete details are illustrated in the following sub sections;

\subsection{DWT based adaptive soft thresholding}

In this subsection, the details of first phase are illustrated completely. Initially the noise contaminated ECG signal is processed through Discrete Wavelet transform to decompose it into approximate and detailed sub bands. Though the Discrete Fourier transform provides a significant signal representation both in phase and amplitude with respect to its frequency, the constant bandwidth of DFT is replaced by DWT through the proportional frequencies by which an effective resolution is obtained for higher frequencies of the signal. The loss in the frequency resolution with the increment in the frequency is acceptable in the applications oriented to bio-medical signals like ECG. Here the DWT based soft thresholding is carried out in three stages, decomposition of noise contaminated ECG signal, adaptive soft thresholding of detailed sub band and reconstruction of denoised subbands.

\subsubsection{DWT based decomposition}

Let $y(n)$ be a noise contaminated ECG signal which needs to be processed for noise filtering, represented as

$$
y(n)=x(n)+v(n)
$$

Where $x(n)$ is the original clean ECG signal and $v(n)$ is the additive noise. Since the ECG signal is one-dimensional signal, the one-dimensional DWT is applied over $y(n)$ to decompose it into approximate and detailed subbands. If $\mathrm{W}$ denotes the wavelet transformation matrix, the Eq. (1) can be represented as

$$
Y=X+V
$$

Where $X=W x(n), V=W v(n)$ and $Y=W y(n)$. A simple architecture of DWT processing is shown in Fig. 1.

\subsubsection{Adaptive soft thresholding}

Compared with time domain analysis, the transformation domain analysis gives more consistent results in the signal analysis. In transformation domain, the signal enhancement approaches which aims to filter out the noise parts form a signal uses amplitude subtraction based soft thresholding, represented as;

$$
\tilde{Y}_{k}= \begin{cases}\left(\left|Y_{k}\right|-\sigma_{v}\right), & \text { if } Y_{k} \geq \sigma_{v} \\ 0, & \text { Otherwise }\end{cases}
$$


Where $\sigma_{v}$ denotes the noise level, $Y_{k}$ is the $\mathrm{k}_{\text {th }}$ coefficient of noisy ECG signal obtained by the transformation analysis and $\tilde{Y}_{k}$ denotes the respective thresholded coefficient. According to the Eq. (3), all the coefficients are subtracted form a constant threshold limit by which the ECG signal components are also discarded. This degradation results in the loss of the signal quality. Hence to overcome this problem with conventional approach, this approach proposes a new thresholding mechanism based on variances. Unlike the conventional constant subtraction rule in Eq. (3), a frame based thresholding strategy is proposed here. This strategy depends on segmenting the signal into short time frames after transforming through DWT. Here the output of DWT is approximate subband and detailed subband. Since the approximate DWT subband coefficients contains the low frequencies of original ECG signal, where most of the energy exists, this work proposed to exclude the approximate subband coefficients in the proposed thresholding operation based on the variance of every frame which was carried out over the detailed subband coefficients only. Since the ECG signals are non-stationary in nature, applying the hard or soft thresholding with a constant $\sigma_{v}$ value like Eq.(3) is not adoptable to reduce the noise. Hence, a new form of soft thresholding is carried out here in the wavelet domain based on the newly derived threshold value. The proposed adaptive thresholding is represented as,

$$
\tilde{Y}_{k}=\operatorname{sign}\left(Y_{k}\right)\left[\max \left\{0,\left(\left|Y_{k}\right|-\mu_{j}\right)\right\}\right]
$$

Where $\mu_{j}$ is a linear threshold function obtained as,

$$
\mu_{j}=j \frac{\lambda \sigma_{n} N}{\sum_{k=1}^{N} k^{2}}
$$

Where $j$ is the index of sorted $\left|Y_{k}\right|$. Before processing the detailed subband coefficients to the adaptive soft thresholding process, the total detailed subband is segmented into frames and further divided into eight frequency bins. After this every frequency bin is categorized into signal dominant and noise dominant frequency bins based on the average noise power. If the $i_{t h}$ frequency bin fulfills the subsequent inequality;

$$
\frac{1}{N} \sum_{k=1}^{N}\left|Y_{k}^{i}\right|^{2} \geq 2 \sigma_{n}^{2}
$$

Where $\mathrm{N}$ is the total number of coefficients in every frequency bin, $\sigma_{n}^{2}$ is the variance of noise and $Y_{k}^{i}$ is the $k_{t h}$ detailed coefficient of the $i_{t h}$ frequency bin; then the frequency bin is categorized as signal dominant otherwise noise-dominant. Further the noise dominant bins are processed for thresholding through Eq. (4). From the expression shown in Eq. (6), for noise-dominant frequency bins, the estimated average noise power over the complete signal is greater than the added average noise power. Here the product of an arbitrary constant $\lambda$ and variance $\sigma_{n}, \lambda * \sigma_{n}$ denotes the average added noise power for any of the frequency bin.

\subsubsection{Average noise power $\left(2 \sigma_{n}^{2}\right)$}

The limit given in Eq.(6) considers the variances of both ECG signal variance $\sigma_{s}^{2}$ and noise signal variance $\sigma_{n}^{2}$ respectively. For a noise contaminated ECG signal, the variance $\sigma^{2}$ for any frequency bin is represented as,

$$
\sigma^{2}=\sigma_{s}^{2}+\sigma_{n}^{2}+2 \varphi(s, n)
$$

Where $\varphi(s, n)$ is the covariance between the terms of signal and noise. If the relation between signal and noise is independent in nature, then the covariance becomes zero, the Eq. (7) becomes

$$
\sigma^{2}=\sigma_{s}^{2}+\sigma_{n}^{2}
$$

for bin categorization into signal-dominant and noise-dominant. If the signal power is equal to the noise power, then the above expression becomes $\sigma^{2}=2 \sigma_{n}^{2}$. Thus the variance of a signal in case of equal noise and signal power is equal to $2 \sigma_{n}^{2}$. The equal variance of noise and signal contributes the equilibrium state of signal noise power to the noisy signal frame. If such type of power is considered for the derivation of threshold, a perfect categorization would be achieved among the frequency bins. The power of any frame exceeding the threshold value constitutes the signal dominant and the below the threshold constitutes the noise dominance.

\subsubsection{Optimum value of $\lambda$}

The performance of proposed method can be further enhanced by deriving an optimal value for $\lambda$. A simple plot linked to the Output SNR for varying $\lambda$ with input SNR is represented in the following Fig. 2.

According to the Fig. 2, it can be observed that it is better to have a higher $\lambda$ value for low SNR input signals and lower $\lambda$ value for high SNR input signals. Here the optimal value of $\lambda$ can be related with the input SNR and relation is defined as, 


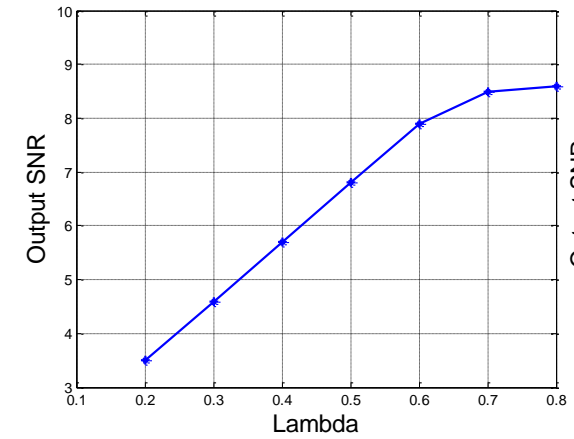

(a)

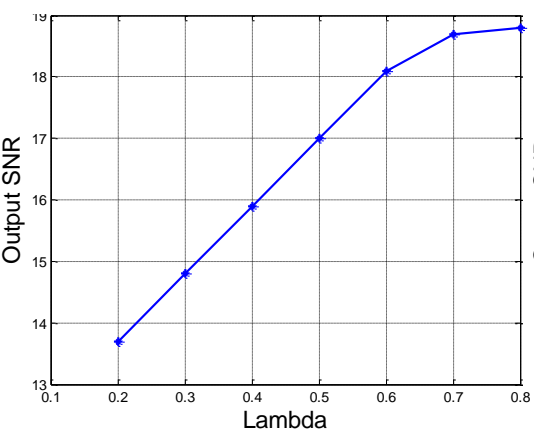

(b)

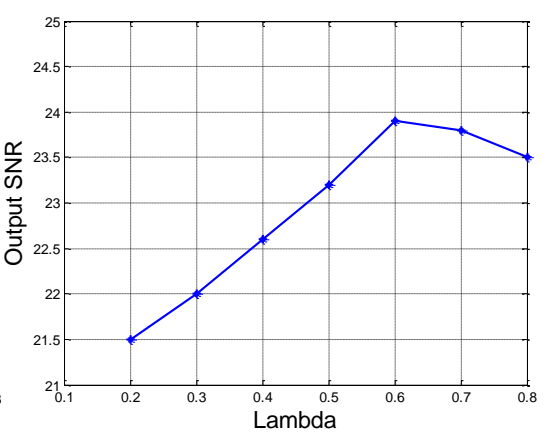

(c)

Figure.2 Effect of lambda on the Output SNRs for: (a) Input SNR 0 dB, (b) Input SNR 10 dB, and (c) Input SNR 20 dB

$$
\lambda_{\text {opt }}=\alpha_{0}-\alpha_{1}\left(S N R_{\text {input }}\right)
$$

Where the $S N R_{\text {input }}$ can be measured as

$$
S N R_{\text {input }}=10 \log \left(\frac{\sigma_{s}^{2}}{\sigma_{n}^{2}}\right)
$$

Where $\sigma_{s}^{2}$ is the signal variance and $\sigma_{n}^{2}$ is the noise variance. $\alpha_{0}$ and $\alpha_{1}$ are the two arbitrary constants which provides a linear relation between the $\lambda_{\text {opt }}$ and input SNR.

Finally the denoised frames are concatenated with signal dominant frames to form denoised detailed subband coefficients and further the inverse DWT is applied over the denoised detailed subband coefficients and unmodified approximate coefficients to reconstruct a noise free ECG signal.

\subsection{EMD based denoising}

After obtaining a denoised ECG signal through first phase DWT based soft thresholding, the second phase applies EMD based denoising to further remove the residual noise components from the first phase output and to preserve the QRS complexes. The basic principle of EMD is to filter out the noise components from the noisy signal by decomposing it into $\mathrm{M}$ number of intrinsic mode functions. However the main problem in EMD based denoising is the appropriate selection of number of IMFs which constitutes only the required information. Various approaches are proposed in earlier to choose the number of IMFs based on various strategies and most of the approaches noticed that and suggested that the most of noise information is present in the initial IMFs and thereby eliminating the possible initial IMFs results in an noise free signal. However the removal initial IMFs results in the loss of QRS complex information which follows the noise statistics. Hence the initial IMFs are also has to be included in the denoising procedure. Instead of removing the initial IMFs completely, this approach tries to remove the noise information from the initial IMFs, results in a noise free and qualitative signal.

Unlike the conventional approaches which tends to perform ECG signal denoising based on the EMD by discarding the initial IMFs containing the QRS complex and noise completely, this approach aimed to preserve the QRS complex information in the first three IMFs by reducing the noise components from them. Compared with the other parts of ECG signal, the rate of information variance in QRS complex is observed to be more and this information is mainly embedded in the first three IMFs. Hence there is a need to develop an approach which just filters out the noise components in the first three IMFs instead of discarding them completely thus preserves the QRS complex information. This can be achieved by temporal processing in the EMD domain. With this objective, the first IMFs are analyzed carefully by summing them and formulating a cumulative signal as $d(n)=c_{1}(n)+c_{2}(n)+c_{3}(n)$, where $c_{1}, c_{2}$ and $c_{3}$ are the first three IMFs obtained after EMD. From the cumulative signal, the QRS complex can be detected by applying a windowing technique. Since the maximum amplitude of an ECG signal lies in the R-peak, the detection of R-peak is important and then finding two local minimal of R-peak, one on the right side and another on the left side. The enveloped formulated through these three points constructs a QRS complex. For this purpose, this approach applies a time window $\omega(n)$ to $d(n)$ centered at the fiducial points such that the length of window covers the entire QRS complex. The window size is varied according to the QRS duration that varies among different ECG signals.

For further IMFs, the soft thresholding is applied based on the IMF variance and the remaining noise is filtered out. After preserving the QRS complex through EMD based windowing, the windowed $d(n)$, soft thresholded IMFs and residue are summed up to reconstruct a noise free ECG signal as, 


$$
\tilde{Y}=Y_{1}+Y_{2}+Y_{3}
$$

Where $\tilde{Y}$ is a noise free ECG signal, $Y_{1}$ is windowed $d(n)$,

$$
Y_{1}=\omega(n) \sum_{i=1}^{3} c_{i}(n)
$$

$Y_{2}$ is the sum of soft thresholded IMFs

$$
Y_{2}=\left(\sum_{i=4}^{M=1} c_{i}(n)+r_{M}(n)\right) \omega(n) d(n)
$$

and $Y_{3}$ is the residue.

$$
Y_{3}=\sum_{i=4}^{M=1} c_{i}(n)+r_{M}(n)
$$

Form the above expressions, it can be noticed that analyzing the signal in time domain (EMD) has the advantages of simplicity in interpretation and calculation. Further it can also be noticed that the consistent analysis is only possible with transformed characteristics of the signal. Hence the proposed can applicable for filtering any type of noise in ECG signals.

\section{Simulation results}

In this section, the performance evaluation of proposed approach is illustrated briefly. Number of experiments is carried out using MATLAB software to simulate the proposed approach through various ECG signals. For every simulation, a comparative analysis is also carried out through state-of art methods through some performance metrics.

\subsection{Database details}

In this simulation, the proposed approach is simulated over the ECG signals collected from the most popular Physionet MIT-BIH arrhythmia database [26]. The MIT-BIH Arrhythmia Database contains 48 ECG recording. Each recording of the ECG signal was digitized at 360 samples per second per channel with 11-bit resolution over $10 \mathrm{mV}$ range. In this database, every ECG signal is represented with three types of file. They are Reference annotation file (.atr), Digitized binary file (.dat) and text header file (.hea). The detailed information of respective ECG, i.e., the ECG signal format, Sampling Frequency, Number of samples, Number of ECG leads and their type, Patients history and also the detailed clinical information is stored in the header file. A sample ECG signal is shown in Fig. 3 (a).

\subsection{Case 1: ECG-PLI}

In this case, the original ECG signal is contaminated with power line interference and the proposed approach is applied noise contaminated ECG to filter out the PLI components. For this purpose, a standard ECG signal is considered and then a noise signal. In general, the PLI noise is added in the ECG signal due to the Electromagnetic Field (EMF) of any device placed nearby, Electromagnetic Interference by power line, Improper grounding of ECG machinery etc. The power line Interference is a signal with frequency range of 50 to $60 \mathrm{~Hz}$ and a sampling frequency of $1000 \mathrm{~Hz}$. Here a signal is generated with these characteristics and added to the ECG signal to obtain the PLI noise contaminated ECG signal. Finally, the power line interference is removed using the conventional EMD based thresholding [5], Wavelet soft thresholding [15], EMD wavelet thresholding [25] and proposed approach and the filtered signals are shown in Fig. 3.

\subsection{Case 2: ECG-baseline wander (BW)}

In this case, the ECG signal is contaminated with baseline wandering noise and then the proposed approach is accomplished over the noise contaminated ECG. The baseline wander noise is a low-frequency noise presents in the ECG signal. The baseline wander noise adds to the ECG due to the body movement, respiration and offset voltages in electrodes etc. The approximate frequency of baseline wander noise is less than $1 \mathrm{~Hz}$. Here Baseline Wander is generated by low-pass filtering of a Gaussian noise of the variance equal to the ECG signal power and then added to the original ECG signal. Further the proposed approach is accomplished over the corrupted ECG signal to remove the baseline wander. The obtained results are shown in Fig. 4.

\subsection{ECG with EMG noise}

In this case, the ECG signal is contaminated with EMG noise and then the proposed approach is accomplished over the noise contaminated ECG. Here the EMG noise is generated with $10 \%$ amplitude of ECG signals amplitude and a frequency range around $200 \mathrm{~Hz}$. The original ECG signal is added to the EMG noise to formulate an EMG noise contaminated ECG signal and further the proposed approach is accomplished over the corrupted ECG signal to remove the EMG noise. The obtained results are shown in Fig. 5. 


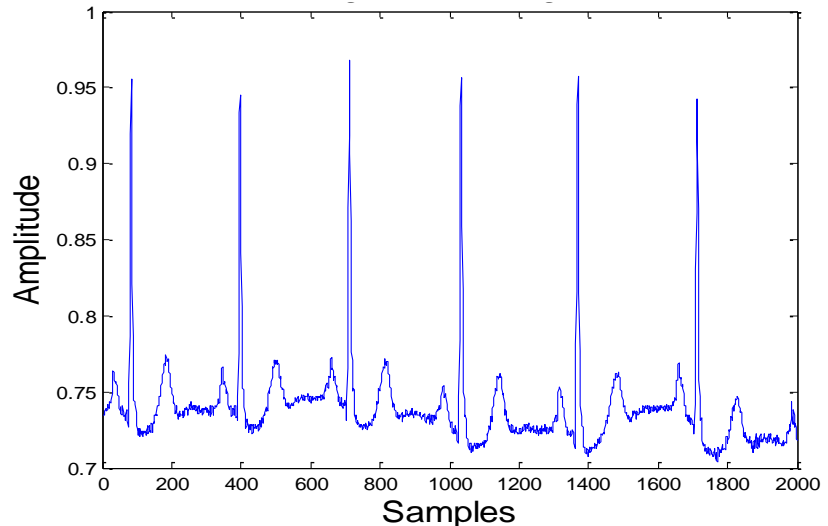

(a)

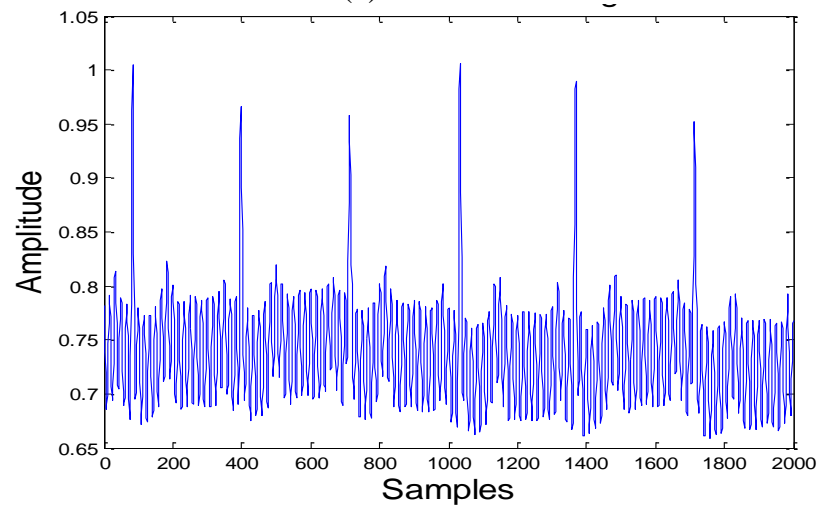

(c)

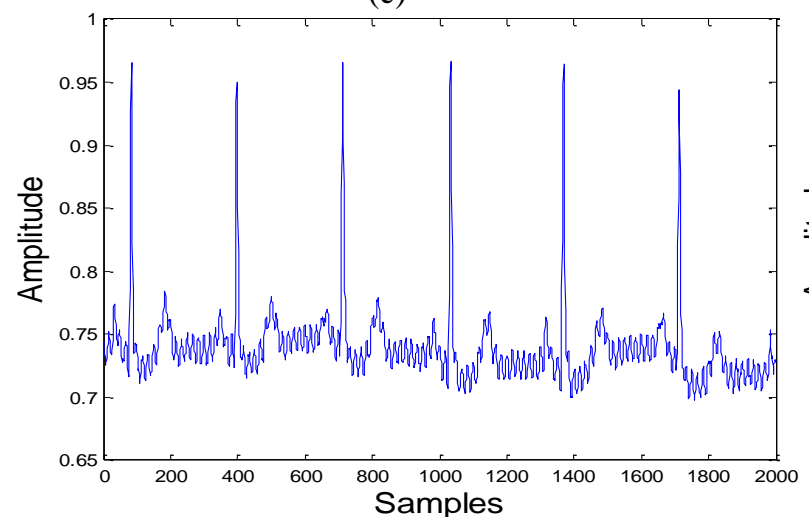

(e)

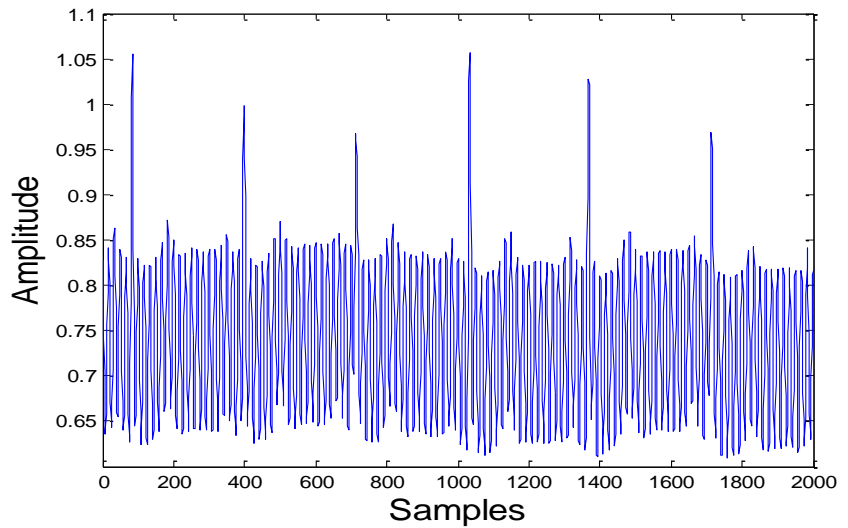

(b)

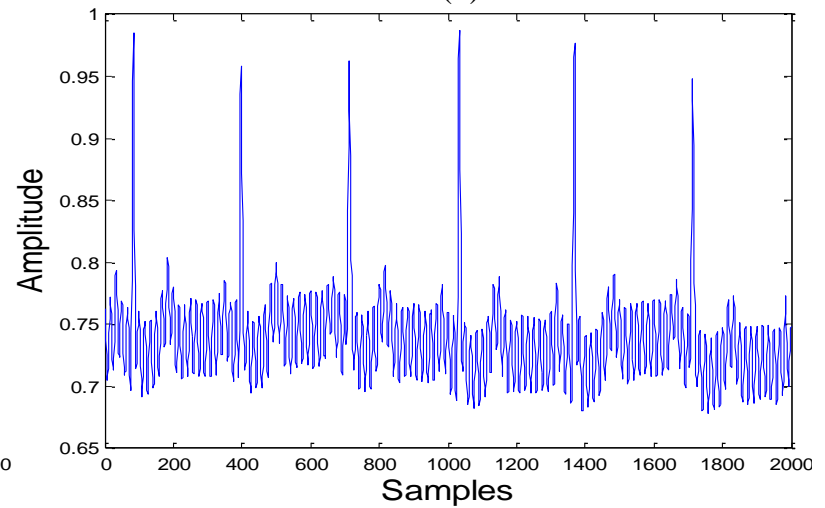

(d)

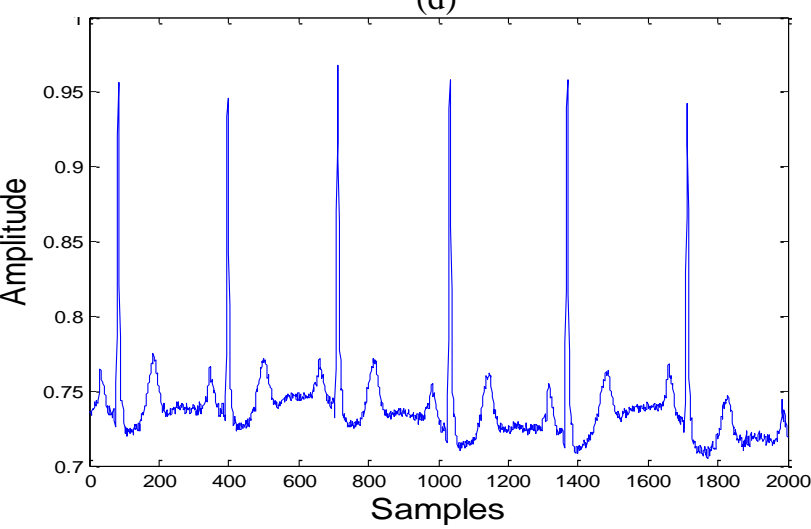

(f)

Figure. 3 Simulation results: (a) Original ECG signal MIT-BIH Arrhythmia Database, (b) Power Line Interference contaminated ECG, (c) EMD based denoising [5], (d) DWT soft thresholding [15], (e) EMD wavelet [25], and (f) Proposed approach

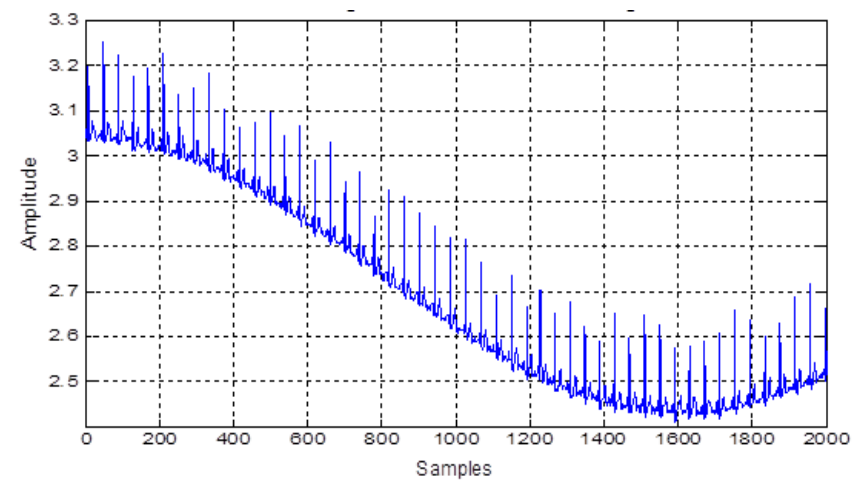

(a)

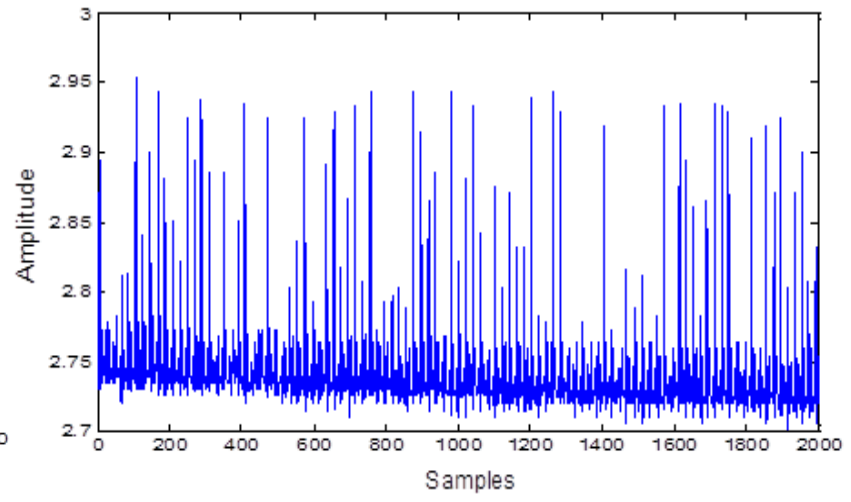

(b)

Figure. 4 Obtained results: (a) ECG signal with baseline wandering and (b) Baseline wander filtered ECG signal 


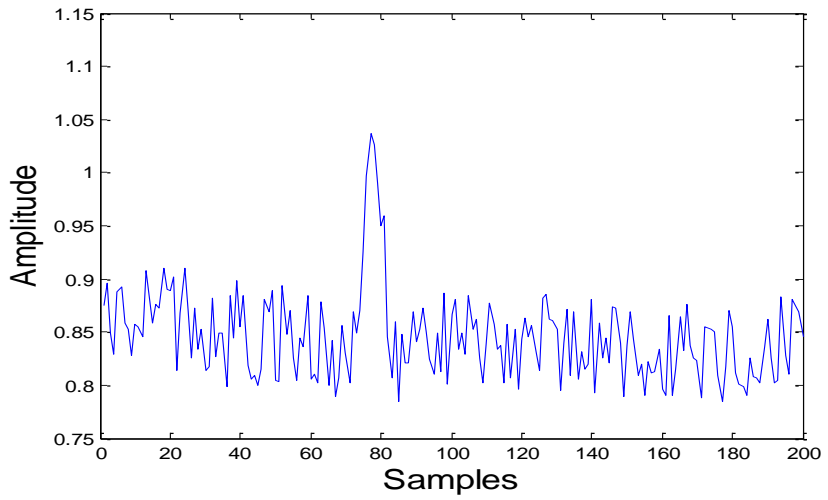

(a)

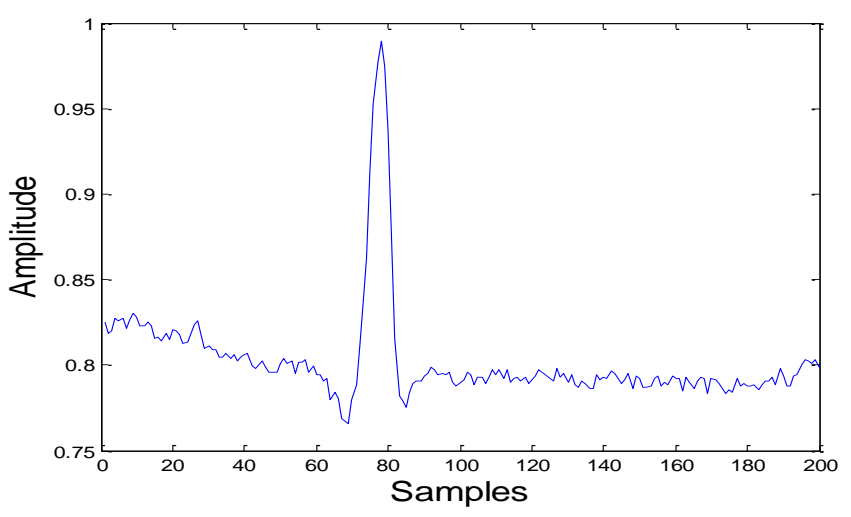

(b)

Figure. 5 Obtained results: (a) ECG signal contaminated with EMG noise and (b) Filtered ECG

Table.1 MSE comparison between different denoising approaches

\begin{tabular}{|c|c|c|c|c|c|}
\hline $\begin{array}{c}\text { Noise } \\
\text { type }\end{array}$ & Signal & $\begin{array}{c}\text { EMD soft } \\
\text { thresholding [5] }\end{array}$ & $\begin{array}{c}\text { Wavelet Soft } \\
\text { Thresholding [15] }\end{array}$ & EMD-Wavelet [25] & Proposed \\
\hline \multirow{2}{*}{ PLI } & S1 & 0.00442 & 0.00382 & 0.00355 & 0.00260 \\
\cline { 2 - 6 } & S2 & 0.00371 & 0.00314 & 0.00285 & 0.00201 \\
\cline { 2 - 6 } & S3 & 0.00323 & 0.00268 & 0.00236 & 0.00197 \\
\hline \multirow{2}{*}{ BW } & S1 & 0.00934 & 0.00877 & 0.00847 & 0.00535 \\
\cline { 2 - 6 } & S2 & 0.00752 & 0.00696 & 0.00669 & 0.00403 \\
\cline { 2 - 6 } & S3 & 0.00805 & 0.00744 & 0.00715 & 0.00454 \\
\hline \multirow{2}{*}{ EMG } & S1 & 0.00606 & 0.00542 & 0.00518 & 0.00425 \\
\cline { 2 - 6 } & S2 & 0.00524 & 0.00463 & 0.00437 & 0.00366 \\
\cline { 2 - 6 } & S3 & 0.00753 & 0.00699 & 0.00669 & 0.00514 \\
\hline
\end{tabular}

\subsection{Performance evaluation}

Under this section, the performance of proposed approach is measured through three performance metrics. They are Signal-to-noise ratio improvement $\left(S N R_{\text {imp }}\right)$, Mean Square Error (MSE) and percent root mean square difference (PRD). The mathematical representations of these performance metrics are given as,

$$
S N R_{i m p}=10 \log _{10} \frac{\sum_{i=1}^{N}|y(i)-x(i)|^{2}}{\sum_{i=1}^{N}|\tilde{x}(i)-x(i)|^{2}}
$$

Where $x(i)$ denotes the original ECG signal, $y(i)$ denotes the noise contaminated ECG signal and $\tilde{x}(i)$ denotes the enhanced ECG signal. The $\mathrm{N}$ represents total number of samples in the signal. Further the MSE and PRD are represented as,

$$
\begin{aligned}
& M S E=\frac{1}{N} \sum_{i=1}^{N}(\tilde{x}(i)-x(i))^{2} \\
& P R D=\sqrt{\frac{\sum_{i=1}^{N}(\tilde{x}(i)-x(i))^{2}}{\sum_{i=1}^{N} x^{2}(i)}} \times 100
\end{aligned}
$$

The performance enhancement achieved in suppressing the unnecessary noise components from the ECG signal can be described through these performance metrics. A method which achieves higher $S N R_{\text {imp }}$ and lower MSE and PRD is said to be an efficient method. The performance metrics are measured for every simulation and compared with the conventional approaches. The obtained MSE details for different simulation through different denoising approaches are illustrated in Table 1.

Table 1 illustrates the details of comparative analysis of the MSE results obtained through the proposed and conventional denoising approaches for the same group of ECG signals at different types of noises. Here the proposed approach is observed to achieve an efficient MSE results compared to the conventional approaches. The less MSE yields in the better quality of signal. Here the MSE is evaluated between the original ECG signal and enhanced ECG signal and as much as the error is less between the original and recovered, the approach is said to be more efficient. Further it is also observed that the proposed approach achieved an efficient MSE result for all types of noise and this achievement indicates the robustness of proposed technique.Since the proposed approach accomplished the denoising at two stages, the residual noise which still exists in the filtered signal after first stage is filtered at second 


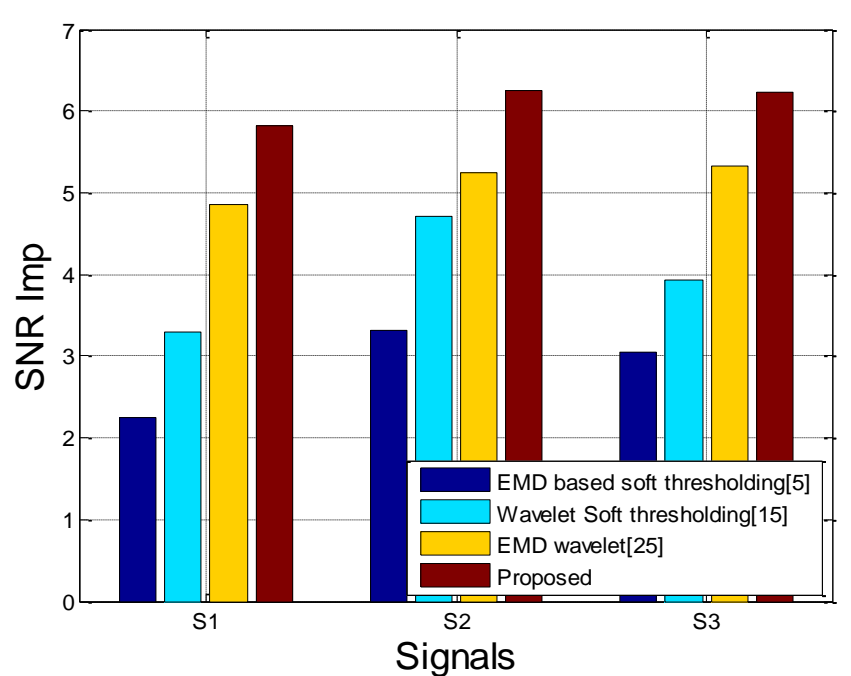

(a)

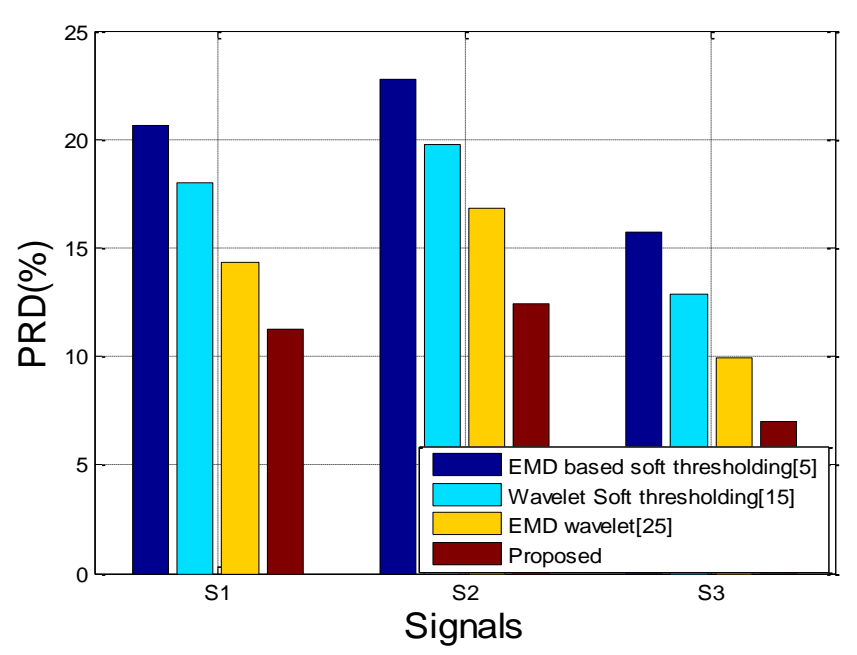

(b)

Figure.6 Comparison of: (a)SNR $R_{\text {imp }}$ and (b) PRD (\%) obtained through different denoising approaches

stage. Almost the complete noise is eliminated in the first stage due to the adaptive thresholding. The proposed adaptive thresholding removes the noise and also preserves the required signal information. Compared to the conventional approaches, the proposed approach is more robust in the reduction of noise which reducesthe MSE between the original and filtered signal. In the EMD based soft thresholding, the noise is assumed to be exists mostly in the initial IMFs and neglects remaining IMFs. Due to this reason, there exists still some more noise in the filtered ECG signal. Further the DWT based soft thresholding also fails in the removal of overall noise.

Further the obtained improvements in the $S N R_{\text {imp }}$ and PRD are represented in the Fig. 6 (a) and (b) respectively.

\section{Conclusions and future scope}

In this paper, a new ECG signal denoising approach is proposed to filter out the unnecessary noise components which add into the ECG signal during its recording. Since the existence of extra noise part in the ECG signal results in a wrong diagnosis, this approach focused to remove the all types of noise like PLI, BW and EMG noises form the ECG signal and making the signal noise free. For this purpose, the filtering is carried out in two phases; the first phase accomplished DWT based soft thresholding followed by EMD based denoising in the second phase. A new formulation to derive the threshold makes the approach more robust to all types of noises and also helps to increase the quality of signal. Performance evaluation is carried out over different ECG signals with different noises and the performance acquisition is measured through the performance metrics MSE, PRD and $S N R_{\text {imp }}$. A comparative analysis is also carried out between the proposed and conventional approached and observed that the proposed approach is better compared to conventional approaches and concluded to be a robust and efficient technique for ECG signal denoising.

The main aim of this paper is to remove the unwanted noises form ECG signal such that the obtained ECG signal makes the automatic CVDs detection system more accurate in the detection of cardiac arrhythmia. For an automatic CA detection system, the features of ECG are more important and the information represented by these feature is more accurate only when the signal is noise free. Thus the future accomplishment of this work is carried out to extract the sufficient and important features such that the automatic CA detection system will be more accurate with less computational overhead.

\section{References}

[1] World Health Organization, "Cardiovascular disease", 2013.

[2] World Health Organization, "From burden to "best buys": reducing the economic impact of non-communicable disease in low-and middle-income countries", Program on the Global Demography of Aging, 2011.

[3] D. W. Smith, D. Nowacki, and J. K. Li, "ECG $\mathrm{T}$-wave monitor for potential early detection and diagnosis of cardiac arrhythmias", Cardiovascular Engineering, Vol. 10, No. 4, pp.201-206, 2010. 
[4] M. Benmaiek, A. Charef, and F. Abdelliche, "Preprocessing of the ECG Signals Using the His-Purkinje Fractal System", In: Proc. of the 7th International Multi-Conf. on Systems, Signals and Devices, pp.1-5, 2010.

[5] K. M. Chang and S. H. Liu, "Gaussian Noise Filtering from ECG by Wiener Filter and Ensemble Empirical Mode Decomposition", Journal of Signal Processing Systems, Vol.64, No.2,pp. 1-16, 2010.

[6] M.P. Chawla, H.K. Verma, and V. Kumar, "Artifacts and noise removal in electrocardiograms using independent component analysis", International Journal of Cardiology, Vol. 129, No.2, pp. 278-281, 2008.

[7] M. Chawan, R. Agarwala, and M.D. Upalane, "Suppression of noise in the ECG signal using digital IIR filter", In: Proc. of the 8th WSEAS International Conf. on Multimedia systems and signal processing, pp.335-343, 2008.

[8] S.M.M. Martens, M. Mischi, S.G. Oei, and J.W.M. Bergmans, "An Improved Adaptive Power Line Interference Canceller for Electrocardiography", IEEE Transactions on Biomedical Engineering, Vol. 53, No.11, pp. $2220-22312006$.

[9] N. Li and P. Li, "An improved algorithm based on EMD-wavelet for ECG signaldenoising", In: Proc. of International Joint Conf. on Computational Sciences andOptimization, pp. 825-827, 2009.

[10] L. N. Sharma, S. Dandapat, and A. Mahanta, "Multiscale principal component analysis to de-noise multichannel ECG signals", In: Proc. of International Conf. on Biomedical Engineering, pp.17-20, 2010.

[11] M.M.V. Hualsaqui, E.I.P. Vizcaino, M.J. Flores Calero, and E.V. Carrera, "ECG signal denoising through kernel principal components", In: Proc. of International Conf. on Electronics, Electrical Engineering and Computing, pp.1-5, 2017.

[12] A.S. Barhatte, R. Ghongade, and S.V. Tekale, "Noise analysis of ECG signal using fast ICA", In: Proc. of International Conf. on Advances in Signal Processing, pp.118$122,2016$.

[13] G. Tang and A. Qin, "ECG de-noising based on empirical mode decomposition", In: Proc. of $9^{\text {th }}$ International Conf. for Young Computer Scientists, pp. 903-906, 2008.

[14] M.A. Alwal and S.S. Mostafa, "An adaptive level dependent wavelet thresholding for ECGdenoising", Bio-cybernetics and
Biomedical Engineering, Vol.34, No.4, pp.238-249, 2014.

[15] A. Milchevski and M. Gusev, "Improved pipelined wavelet implementation for filtering ECG signals", Pattern Recognition Letters, Vol. 95, No.1, pp. 85-90, 2017.

[16] W. Jenkal, R. Latif, and A. Toumanari, "An efficient algorithm of ECG signal denoising using the adaptive dual threshold filter and the discrete wavelet transform", Bio-cybernetics and Biomedical Engineering,Vol.36, No.3, pp.499-508, 2016.

[17] Y. Kopsinis and S. McLaughlin, "Development of EMD-based denoising methods inspired by wavelet thresholding", IEEE Transactions on Signal Processing, Vol.57, No.4, pp.1351-1362, 2009.

[18] M.A. Kabir and C. Shahanaz, "Denoising of ECG signals based on noise reduction algorithms in EMD and wavelet domains", Biomedical Signal Processing and Control, Vol.7, No.5, pp. 481-489, 2012.

[19] H. Kasturiwale and C. Deshmukh, "Quality assessment of ICA for ECG signal analysis", In: Proc. of the 2nd International Conf. on Emerging Trends inEngineering and Technology, pp. 73-75, 2009.

[20] R. Sameni, M. Shamsollahi, C. Jutten, and G. Clifford, "A nonlinear Bayesian filtering framework for ECG denoising", IEEE Transactions on Biomedical Engineering, Vol. 54, No.12, pp. 2172-2185, 2007.

[21] L. Smital, M. V'itek, J. Kozumpl'ik, and I. Provazn'1k, "Adaptive Wavelet Wiener Filtering of ECG Signals", IEEE Transactions on Biomedical Engineering, Vol. 60, No. 2, pp. 437-445, 2013.

[22] H. Choi and R. Baraniuk, "Analysis of wavelet-domain Wiener filters", In: Proc. of the IEEE International Symp. on TimeFrequency Time-Scale Analysis, pp. 613-616, 1998.

[23] A. Phinyomark, C. Limsakul, and P. Phukpattaranont, "EMG denoisingestimation based on adaptive wavelet thresholding for multifunction myoelectriccontrol", In: Proc. Innovative Technol. Intell. Syst. Ind. Appl., pp.171-176, 2009.

[24] M. Jansen, "Noise Reduction by Wavelet Thresholding", eBook, Springer-Verlag, New York, 2001.

[25] P. Singh and G. Pardhan, "Denoising of ECG signal by non-local estimation of approximation coefficients in DWT", Bio- 
cybernetics and Biomedical Engineering, Vol.37, No. 3, pp.599-610, 2017.

[26] G.B. Moody and R.G. Mark, "The impact of MIT-BIH Arrhythmia Database", IEEE Transaction on Engineering in Medicine and Biology, Vol.20, No.3, pp.45-50, 2001. 